\title{
White matter extension of the Melbourne Children's Regional Infant Brain atlas: M-CRIB-WM
}

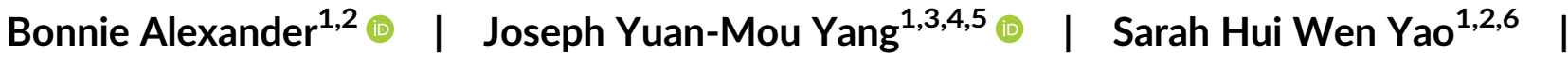 \\ Michelle Hao Wu $\mathbf{u}^{7}$ Jian Chen ${ }^{1,8}$ | Claire E. Kelly ${ }^{1,2}$ ( ) | Gareth Ball ${ }^{1}$ ( ) | \\ Lillian G. Matthews ${ }^{1,2,9}$ | Marc L. Seal ${ }^{1,5}$ | Peter J. Anderson ${ }^{2,10}$ | \\ Lex W. Doyle $e^{2,5,11,12}$ | Jeanie L. Y. Cheong $2,11,12$ | Alicia J.Spittle $2,11,13$ | \\ Deanne K. Thompson ${ }^{1,2,5,14}$
}

${ }^{1}$ Developmental Imaging, Murdoch Children's Research Institute, Melbourne, Victoria, Australia

${ }^{2}$ Victorian Infant Brain Studies, Murdoch Children's Research Institute, Melbourne, Victoria, Australia

${ }^{3}$ Neuroscience Research, Murdoch Children's Research Institute, Melbourne, Victoria, Australia

${ }^{4}$ Department of Neurosurgery, Royal Children's Hospital, Melbourne, Victoria, Australia

${ }^{5}$ Department of Paediatrics, The University of Melbourne, Melbourne, Victoria, Australia

${ }^{6}$ Monash School of Medicine, Faculty of Medicine, Nursing and Health Sciences, Monash University, Clayton, Victoria, Australia

${ }^{7}$ Medical Imaging, Royal Children's Hospital, Melbourne, Victoria, Australia

${ }^{8}$ Department of Medicine, Monash University, Melbourne, Victoria, Australia

${ }^{9}$ Department of Pediatric Newborn Medicine, Brigham and Women's Hospital, Harvard Medical School, Boston, Massachusetts

${ }^{10}$ Turner Institute for Brain and Mental Health, School of Psychological Sciences, Monash University, Melbourne, Victoria, Australia

${ }^{11}$ Newborn research, Royal Women's Hospital, Melbourne, Victoria, Australia

${ }^{12}$ Department of Obstetrics and Gynaecology, The University of Melbourne, Melbourne, Victoria, Australia

${ }^{13}$ Department of Physiotherapy, The University of Melbourne, Melbourne, Victoria, Australia

${ }^{14}$ Florey Institute of Neuroscience and Mental Health, Melbourne, Victoria, Australia

\section{Correspondence}

Deanne K. Thompson, Murdoch Children's Research Institute, Royal Children's Hospital, Flemington Road, Parkville, Victoria 3052, Australia.

Email: deanne.thompson@mcri.edu.au

Funding information

University of Melbourne; Royal Children's Hospital Foundation; National Health and

Medical Research Council, Grant/Award

\begin{abstract}
Brain atlases providing standardised identification of neonatal brain regions are key in investigating neurological disorders of early childhood. Our previously developed Melbourne Children's Regional Infant Brain (M-CRIB) and M-CRIB 2.0 neonatal brain atlases provide standardised parcellation of 100 brain regions including cortical, subcortical, and cerebellar regions. The aim of this study was to extend M-CRIB atlas coverage to include 54 white matter (WM) regions. Participants were 10 healthy
\end{abstract}

Abbreviations: AAL, automated anatomical labeling; ACR, anterior corona radiata; ALIC, anterior limb of internal capsule; ANTs, advanced normalization tools; AP, anterior-posterior; BET, brain extraction tool; $\mathrm{CC}$, corpus callosum; $\mathrm{CGC}$, cingulum cingular part; $\mathrm{CGH}$, cingulum hippocampal part; $\mathrm{CP}$, cerebellar peduncle; $\mathrm{CR}$, corona radiata; CST, corticospinal tracts; $\mathrm{DEC}$, directionencoded color; DTI, diffusion tensor imaging; DWI, diffusion weighted images; EC, external capsule; EPI, echo planar imaging; FLIRT, Functional Magnetic Resonance Imaging of the Brain's Linear Image Registration Tool; FMRI, functional magnetic resonance imaging; FOV, field of view; FSL, functional magnetic resonance imaging Software Library; FUGUE, FMRIB's utility for geometrically unwarping echo planar images; Fx, fornix; GM, grey matter; IC, internal capsule; ICP, inferior cerebellar peduncle; IFO, inferior fronto-occipital fasciculus; ITK, Insight Toolkit; JHU, Johns Hopkins University; LR, left-right; MCP, middle cerebellar peduncle; M-CRIB, Melbourne Children's Regional Infant Brain; M-CRIB-WM, Melbourne Children's Regional Infant Brain-white matter; ML, medial lemniscus; MRI, magnetic resonance imaging; PCR, posterior corona radiata; PCT, pontine crossing tract; PLIC, posterior limb of internal capsule; PTR, posterior thalamic radiation; RLIC, retrolenticular part of internal capsule; SCP, superior cerebellar peduncle; SCR, superior corona radiata; SFO, superior fronto-occipital fasciculus; SI, superior-inferior; SLF, superior longitudinal fasciculus; SS, sagittal stratum; ST, stria terminalis; TAP, tapetum; TE, echo time; TR, repetition time; UFC, uncinate fasciculus; WM, white matter.

This is an open access article under the terms of the Creative Commons Attribution License, which permits use, distribution and reproduction in any medium, provided the original work is properly cited.

(c) 2020 The Authors. Human Brain Mapping published by Wiley Periodicals, Inc. 
Numbers: $1024516,1028822,1153176$, 1060733, 546519, 1081288, 1053787, 1053767, 1012236, 1108714, 1085754, 1160003 term-born neonates that were used to create the initial M-CRIB atlas. WM regions were manually segmented based on $T_{2}$ images and co-registered diffusion tensor imaging-based, direction-encoded colour maps. Our labelled regions imitate the Johns Hopkins University neonatal atlas, with minor anatomical modifications. All segmentations were reviewed and approved by a paediatric radiologist and a neurosurgery research fellow for anatomical accuracy. The resulting neonatal WM atlas comprises 54 WM regions: 24 paired regions, and six unpaired regions comprising five corpus callosum subdivisions, and one pontine crossing tract. Detailed protocols for manual WM parcellations are provided, and the M-CRIB-WM atlas is presented together with the existing M-CRIB cortical, subcortical, and cerebellar parcellations in 10 individual neonatal MRI data sets. The novel M-CRIB-WM atlas, along with the M-CRIB cortical and subcortical atlases, provide neonatal whole brain MRI coverage in the first multi-subject manually parcellated neonatal atlas compatible with atlases commonly used at older time points. The M-CRIB-WM atlas is publicly available, providing a valuable tool that will help facilitate neuroimaging research into neonatal brain development in both healthy and diseased states.

\section{KEYWORDS}

atlas, MRI, neonatal, parcellation, white matter

\section{1 | INTRODUCTION}

Parcellated brain atlases are a key component of many neuroimaging tools. They can facilitate identification and labelling of brain regions in a consistent manner, such that properties of these regions can be compared across brains and across time points. Until recently, few parcellated atlases were available for the crucial neonatal time period where the foundations for all future neurodevelopment are set. Various perinatal events and conditions, such as very preterm birth, congenital heart disease, neonatal encephalopathy, or stroke, may be associated with alterations to white matter (WM) development and in turn adverse neurodevelopmental outcomes (Anderson, Cheong, \& Thompson, 2015; Dubois et al., 2014). Investigating properties of individual WM regions, such as volume, shape and surface area, microstructure, and myelination, and their behavioural and clinical correlates in typically and atypically developing populations, is therefore of potential clinical relevance (e.g., Mori 2009; Oishi et al., 2009). The provision of WM atlases at the neonatal time point is critical for investigating both typical and atypical WM development.

During the neonatal period, MRI images have relatively low spatial resolution due to small brain size and have different tissue contrast compared with older children and adults due to partial myelination and dynamic tissue properties in neonates (Heemskerk et al., 2013). Over the last decade, increasing efforts in the neonatal brain imaging field have led to development of several neonatal parcellated atlases (Alexander et al., 2017; Alexander et al., 2019; Blesa et al., 2016; de Macedo Rodrigues et al., 2015; Feng et al., 2019; Gousias et al., 2012; Kuklisova-Murgasova et al., 2011; Makropoulos et al., 2016; Oishi et al., 2011; Shi et al., 2010; Shi et al., 2011). These atlases differ in image modality and quality, parcellation technique, and parcellation schemes. Many of these atlases were defined on $T_{2}$-weighted images (which provide higher tissue contrast than $T_{1}$-weighted images due to partial myelination at the neonatal time point) and focus on parcellation of cortical regions and deep grey nuclei. WM segmentation has generally been provided as a single label or a few regions (Alexander et al., 2017; Alexander et al., 2019; de Macedo Rodrigues et al., 2015), or included together with adjacent grey matter (GM) in parcellated regions (Gousias et al., 2012; Shi et al., 2011; Tzourio-Mazoyer et al., 2002). The major WM tracts are extant at term (Dubois et al., 2014), however, these cannot be defined based on $T_{1}$ - or $T_{2}$-weighted images alone. In order to delineate anatomical tracts within WM, diffusion weighted images (DWI), which provide information about WM fibre orientation, are required.

One atlas to date, the 'JHU-neonate-SS' atlas (Oishi et al., 2011) has provided manually delineated anatomical WM regions using neonatal DWI data. The atlas consists of voxel-wise averaging of 122 parcellated brain regions altogether, including $52 \mathrm{WM}$ regions, from the MRI data of 25 healthy neonates. The WM regions were manually segmented based on a single participant's MRI data set, which was then warped to the group-averaged brain template. The parcellated detail of the JHU-neonate-SS atlas is unprecedented and demonstrates the ability of the included regions to be delineated at term. This has allowed properties of these WM regions such as diffusion metrics and connectivity to be studied in neonates (e.g., Chang et al., 2016: Pannek, Hatzigeorgiou, Colditz, \& Rose, 2013). Importantly, the JHU-neonate-SS atlas provides standardised identification of regions at the neonatal time point and compatibility with the adult JHU atlas (Mori et al., 2008), such that regional properties can be 
compared across developmental time points. However, a training set comprising a single participant does not contain individual variability in brain morphology.

Accounting for individual anatomical variance is an important factor to consider when studying a period of brain development that is marked by significant brain structural changes and growth (Shi et al., 2011). Some endeavours have been made to address this issue by warping existing single-subject atlases to multiple neonatal subjects, with the aim of providing larger atlas training sets with greater intersubject variability. For example, Shi et al. (Shi et al., 2011) warped the adult parcellated 'Automated Anatomical Labelling' (AAL) atlas (Tzourio-Mazoyer et al., 2002) (defined based on $T_{1}$ images) to an infant longitudinal sample. However, it is generally acknowledged that warping adult atlases to infant space offers limited accuracy, due to morphological differences between the adult brain and the developing neonatal brain (Alexander et al., 2017; Blesa et al., 2016; Dickie et al., 2017; Fillmore, Richards, Phillips-Meek, Cryer, \& Stevens, 2015; Kazemi, Moghaddam, Grebe, Gondry-Jouet, \& Wallois, 2007; Richards, Sanchez, Phillips-Meek, \& Xie, 2016; Sanchez, Richards, \& Almli, 2012). Additionally, warping a single parcellated image to multiple participants, even those of the same age, is likely to introduce labelling error related to imperfect registration aligning different target brains. This occurs in instances where there are individual differences in morphology or image properties between the template and the target brains (Akhondi-Asl, Hoyte, Lockhart, \& Warfield, 2014). Target brains that differ more greatly from the template will incur more marked registration error, and thus a multi-subject training set that captures some of the individual variability in the population is valuable. The 'gold standard' procedure for defining an accurate and broadly applicable parcellated atlas is manual segmentation in a large sample of representative individuals (Gousias et al., 2012; Shi et al., 2010). In adults, training sets comprising 10 individuals have been found sufficient to optimise parcellation based on label fusion algorithms (Heckemann, Hajnal, Aljabar, Rueckert, \& Hammers, 2006) and optimise structural templates to represent morphological variability in the population (Croxson, Forkel, Cerliani, \& Thiebaut de Schotten, 2018); with additional subjects providing diminishing (Heckemann et al., 2006) or no (Croxson et al., 2018) benefit. Although equivalent studies do not yet appear available in neonates, this provides a baseline indication of sample size from which to begin in neonatal data, while acknowledging the potentially greater variability in morphology observed at this time point.

We previously presented the Melbourne Children's Regional Infant Brain (M-CRIB) atlas, a multi-subject ( $N=10)$, manually parcellated atlas of cortical, basal ganglia, thalamic, cerebellar, and other subcortical regions (Alexander et al., 2017) that is compatible with the Desikan-Killiany adult cortical atlas (Desikan et al., 2006) and some subcortical regions automatically segmented in FreeSurfer. Compatibility of neonatal atlases with those commonly used at older timepoints is important for longitudinal investigations of brain development and for tracking the progression of developmental disorders (de Macedo Rodrigues et al., 2015; Gousias et al., 2012; Oishi et al., 2011). There is currently an unmet need for a multi-subject, manually parcellated neonatal WM atlas to provide standardised identification of WM regions in a way that is compatible with atlases commonly used at older time points. The aim of this study was to extend the coverage of the M-CRIB atlas to include manually parcellated WM regions by utilising neonatal DWI data. We elected to model our parcellation scheme on that provided by the JHU-neonate-SS atlas (Oishi et al., 2011), which has label nomenclature and terminology consistent with the commonly used adult JHU atlas (Mori et al., 2008; Mori, W., Nagae-Poetscher, \& van Zijl, 2005; Oishi et al., 2009). In this article, we detail a manual WM parcellation scheme in 10 term-born neonates, presented as a WM extension of the M-CRIB atlas, which we have named the M-CRIB-WM atlas. Together with the M-CRIB cortical and subcortical atlases, we provide the first detailed multi-subject neonatal atlas encompassing whole brain MRI coverage, including extensive standardised GM and WM parcellations.

\section{MATERIALS AND METHODS}

\section{1 | Participants}

Participants were 10 healthy term-born neonates ( $\geq 37$ weeks' gestation; four females; gestational age at scanning 40.29-43.00 weeks, $M=41.71, S D=1.31$ ). These participants were the same as those utilised for our existing M-CRIB atlases (Alexander et al., 2017; Alexander et al., 2019). The participants were initially selected from a larger cohort of control infants with MRI scans, recruited as part of preterm studies (Spittle et al., 2014; Walsh, Doyle, Anderson, Lee, \& Cheong, 2014). The 10 images included in this study were chosen on the basis of minimal motion and other artefact on $T_{2}$ - weighted images (Alexander et al., 2017). Neonates who received resuscitation at birth, were admitted to a neonatal intensive care or special care unit, had a birth weight of less than $2.5 \mathrm{~kg}$, or had congenital conditions affecting growth and development were excluded (Spittle et al., 2014; Walsh et al., 2014). All 10 participants selected were assessed at age 2 years and did not have any major health problems, cerebral palsy or major cognitive delay (Alexander et al., 2017).

This study was approved by the Royal Children's Hospital Human Research Ethics Committees. Informed parental/guardian consent was obtained prior to the study commencement.

\section{2 | MRI data acquisition and preprocessing}

Neonatal MRI scans were acquired at the Royal Children's Hospital, Melbourne, Australia, on a 3-Tesla Siemens MAGNETOM Trio Tim scanner. Participants were scanned during nonsedated natural sleep. They were first fed, swaddled and fitted with ear plugs and ear muffs throughout the MRI study. Transverse $T_{2}$ restore turbo spin echo sequences were acquired with $1 \mathrm{~mm}$ axial slices, flip angle $=120^{\circ}$, repetition time $(T R)=8,910 \mathrm{~ms}$, echo time $(T E)=152 \mathrm{~ms}$, field of view $($ FOV $)=192 \times 192 \mathrm{~mm}^{2}$, matrix $=384 \times 384$, and in-plane resolution $1 \mathrm{~mm}^{2}$ (automatically zero-filled interpolated in image reconstruction to 
$0.5 \times 0.5 \times 1 \mathrm{~mm}$ ). DWI sequences were acquired using a multi- $b$ value, single-shot echo planar imaging (EPI) sequence with $\mathrm{TR}=20,400 \mathrm{~ms}, \mathrm{TE}=120 \mathrm{~ms}$, FOV $=173 \times 173 \mathrm{~mm}^{2}$, matrix $=$ $144 \times 144,100$ axial slices, $1.2 \mathrm{~mm}$ isotropic voxels, 45 noncollinear gradient directions, $b$-values ranging from 100 to $1,200 \mathrm{~s} / \mathrm{mm}^{2}$, and $3 \mathrm{~b}=0 \mathrm{~s} / \mathrm{mm}^{2}$ volumes. The total diffusion sequence was divided into three separate acquisitions to improve compliance, and if any of the diffusion acquisitions had unacceptable levels of motion artefact, the scan was repeated whenever possible until acceptable diffusion images were acquired. All infants were scanned with the same diffusion sequence, including the same range of $b$-values.

The $T_{2}$ images were preprocessed prior to manual segmentation of the original M-CRIB atlas (Alexander et al., 2017; Loh et al., 2016) and did not undergo any further preprocessing here. For the existing preprocessing, images were bias corrected using N4ITK (http://www. itk.org, RRID:SCR_001149) (Tustison et al., 2010), and skull-stripped using the Functional MRI of the Brain (FMRIB) Software Library (FSL; http://www.fmrib.ox.ac.uk/fsl/, RRID:SCR_002823) Brain Extraction Tool (BET) (Smith, 2002). They were then aligned to anterior commissure-posterior commissure line using 3D Slicer to correct for any head tilt, which could adversely affect delineation, thus allowing equivalent viewing perspectives of structures bilaterally; and resampled to $0.63 \mathrm{~mm}$ isotropic (calculated as the cube root of the $0.5 \times 0.5 \times 1$ voxel size in order to obtain isotropic voxels while preserving voxel volume) using the FMRIB's Linear Image Registration Tool (FLIRT) (Greve \& Fischl, 2009; Jenkinson, Bannister, Brady, \& Smith, 2002; Jenkinson \& Smith, 2001).

The DWI data were corrected for head motion and eddy current-induced distortions using the FSL "eddy_correct" tool (Jenkinson \& Smith, 2001), incorporating b-vector reorientation (Leemans \& Jones, 2009). Echo planar image distortions were corrected based on a gradient echo field map and FMRIB's Utility for Geometrically Unwarping Echo planar images (FUGUE), as previously described (Thompson et al., 2018). The diffusion tensor imaging (DTI) model was fitted using the weighted linear least squares method in FSL. The $b=0 \mathrm{~s} / \mathrm{mm}^{2}$ images were first linearly registered to the $T_{2}$ image using FLIRT (Greve \& Fischl, 2009; Jenkinson et al., 2002; Jenkinson \& Smith, 2001), followed by nonlinear registration to the same $T_{2}$ image using Advanced Normalisation Tools (ANTs; http://www.picsl.upenn.edu/ANTS/, RRID:SCR_004757) (Avants et al., 2011; Avants, Epstein, Grossman, \& Gee, 2008), with symmetric diffeomorphic normalisation as the transformation type and cross correlation as the similarity metric. The concatenated linear and nonlinear transformation matrices derived from both registration steps were applied to the tensor image (including reorientation of the tensor vectors), using the ANTs "Reorient tensor image" option, with linear interpolation used in resampling. Then, the principal DTI eigenvector (representing the modelled single-fibre orientation per voxel) was multiplied by the fractional anisotropy (FA) image to generate a direction-encoded colour (DEC) map with the default colour scheme: the anterior-posterior (AP)-oriented fibres encoded in green; leftright (LR)-oriented fibres encoded in red; and superior-inferior (SI)oriented fibres encoded in blue.

\section{3 | Protocols for delineating the WM regional boundaries}

We derived our parcellation scheme from the JHU neonatal atlas parcellation scheme (Oishi et al., 2011) with modifications to improve anatomical clarity of the following WM structures. First, we incorporated five corpus callosum (CC) vertical subdivisions based on Hofer' Classification (Hofer \& Frahm, 2006) instead of a LR division, considering the interhemispheric nature of this commissural WM tract. We adopted Hofer's CC subdivision method instead of a three parts (genu, body, and splenium) vertical subdivision for two main reasons. First, the subdivision scheme proposed in Hofer's Classification is based on dividing the maximum AP length of the mid-sagittal CC into specific proportions. This helps standardise our parcellation protocol, making the parcellation more likely to be reproducible between different MRI data sets. Second, Hofer's Classification has previously been applied to processing of neonatal data, including reconstructing CC tractography based on these five subdivisions (Thompson et al., 2011; Thompson et al., 2012). Next, we utilised a LR division for the middle cerebellar peduncle (MCP) and labelled the pontine crossing tract (PCT) as a singular region instead of a LR division. The MCP is anatomically a 'paired' (i.e., left and right) structure connecting the cerebellum to pons. It consists of cerebellar afferent fibres from contralateral pontine nuclei, with fibres lateralised to each cerebellar hemisphere and the cerebellar vermis. Centrally, the PCT label constitutes these crossing pontine fibres, thus a singular label is anatomically more appropriate than a LR division. Lastly, we excluded brainstem divisions in our parcellation scheme, because such regions would be sections containing remaining voxels outside of the existing subregions traced in the brainstem. Thus, they could not be combined to produce complete brainstem subsections, or anatomically meaningful regions. Instead, we used the existing brainstem label already included in the original M-CRIB and M-CRIB 2.0 atlases.

\subsection{1 | Regions in the brainstem}

\section{Corticospinal tracts}

Description: This parcellation defines the portion of the corticospinal tracts (CST) in the ventral pons (Ture, Yasargil, Friedman, \& Al-Mefty, 2000). They consist of SI-oriented fibres (blue-coloured on DEC map). Relevant boundaries: Superior: The midbrain-pontine junction Inferior: The pontine-medullary junction Posterior: The pontine crossing tract (PCT; described below).

Pontine crossing tract

Description: This parcellation consists of LR-oriented fibres from the pontine nuclei in the ventral pons (red colour on DEC map). Relevant boundaries: Superior: The midbrain-pontine junction. Inferior: The pontine-medullary junction. Anterior: The CST (described above). Posterior: The Medial Lemniscus (ML; described below) (Mori et al., 2008). 


\section{Medial lemniscus}

Description: This parcellation defines the portion of $M L$ in the ventral pons. They consist of SI-oriented fibres (blue colour on DEC map) (Mori et al., 2008). Relevant boundaries: Superior: The midbrain-pontine junction. Inferior: The pontine-medullary junction. Anterior: The PCT (described previously).

\section{Superior cerebellar peduncle}

Description: This structure contains efferent cerebellar fibres, connecting the cerebellum to the midbrain. It is most easily distinguished from between the level of the cerebellar nuclei and the midbrain using the DEC map (Mori et al., 2008). Relevant boundaries: Superior: It is marked by the superior cerebellar peduncle (SCP) decussation fibres (red coloured on the colour DEC map) at the level of the midbrain. (Mori et al., 2008). Inferior: The dentate nuclei of the cerebellum, located medio-posteriorly to the middle cerebellar peduncle (MCP; described below).

\section{Middle cerebellar peduncle}

Description: This structure contains entirely afferent cerebellar fibres, connecting the cerebellum to the ventral pons. Relevant boundaries: Posterior: The dentate nuclei in the cerebellum. Medial: The CST, PCT, and $\mathrm{ML}$ parcellations in the ventral pons, best visualised on the axial plane.

\section{Inferior cerebellar peduncle}

Description: This structure contains the spinocerebellar fibre tracts, connecting the cerebellum to the medulla and spinal cord. Relevant boundaries: Superior: The level of the mid-pons. Inferior: The dorsolateral aspect of the medulla (Hirsch et al., 1989).

\subsection{2 $\quad$ Projection Fibres}

The projection fibres enter or exit the brain via the spinal cord by traversing through the following structures - from inferiorly to superiorly (for ascending afferent fibres; or in reverse order for descending efferent fibres): cerebral peduncle (CP), internal capsule (IC), and corona radiata (CR). The CP-IC boundary was arbitrarily defined at the level of the anterior commissure (Mori et al., 2008). The IC-CR boundary was arbitrarily defined at the axial level where the IC and external capsule (EC) merged (Mori et al., 2008). The IC can be identified on axial planes as the 'bend-shaped' WM region located between the caudate nucleus, the lentiform nucleus, and the thalamus. It was arbitrarily divided into four parts: the anterior limb (ALIC), the genu, the posterior limb (PLIC), and the retrolenticular part (RLIC). The CR was arbitrarily divided into three parts: anterior (ACR), superior (SCR), and posterior corona radiata (PCR) (Mori et al., 2008).

\section{Cerebral peduncle}

Description: The IC converges into the $\mathrm{CP}$, forming the ventral portion of the midbrain. Relevant boundaries: Superior: The CP-IC plane at the anterior commissure level (Mori et al., 2008). Inferior: The midbrainpontine junction.

\section{Anterior limb of internal capsule}

Description: The ALIC is the anterior bend of the IC in front of the genu. We included the genu of IC into this parcellation - as per the approach adopted by the JHU-neonatal-SS atlas. Relevant boundaries: Superior: The IC-CR plane against the ACR (Mori et al., 2008). Inferior: The CP-IC plane (Mori et al., 2008). Medial: The head of the caudate nucleus. Lateral: The lentiform nucleus. Posterior: The PLIC (described below).

\section{Posterior limb of internal capsule}

Description: This structure represents the posterior bend of the IC, behind the genu. Relevant boundaries: Superior: The CR-IC plane against the SCR. Inferior: The CP-IC plane. Anterior: The genu of the IC. Posterior: The RLIC. This posterior boundary can be identified by a change in the dominant fibre orientation identified on the DEC map from predominantly blue-coloured SI-oriented fibres in the PLIC to predominantly green-coloured AP-oriented fibres in the retrolenticular part of internal capsule (RLIC; described below). Medial: The thalamus. Lateral: The lentiform nucleus.

\section{Retrolenticular part of internal capsule}

Description: This portion of the IC is caudal to the lenticular nucleus and carries the optic radiation. Relevant boundaries: Superior: The CRIC plane against the PCR. Inferior: The sagittal stratum (SS) at the CPIC plane. Anterior: The PLIC. Posterior: The posterior thalamic radiation (PTR). This posterior boundary was arbitrarily defined by an imaginary vertical line extending from the midpoint of the $\mathrm{CC}$ splenium in the mid-sagittal plane (Mori et al., 2008).

\section{Anterior corona radiata}

Description: The CR refers to the subcortical WM region inferior to the centrum semiovale and superior to the IC. Relevant boundaries: Inferior: The CR-IC plane against the ALIC (Mori et al., 2008). Posterior: The superior corona radiata. This posterior boundary was defined arbitrarily by an imaginary vertical line extending from the posterior edge of the genu of $\mathrm{CC}$ in the mid-sagittal plane (Mori \& van Zijl, 2007).

\section{Superior corona radiata}

Description: Arbitrary boundaries were used to parcellate the SCR. Relevant boundaries: Inferior: The CR-IC plane against the PLIC (described previously). Anterior: The ACR (described previously). Posterior: The PCR. This posterior boundary was defined arbitrarily by an imaginary vertical line extending from the anterior edge of the $\mathrm{CC}$ splenium in the mid-sagittal plane (Mori et al., 2008; Mori \& van Zijl, 2007).

\section{Posterior corona radiata}

Description: Arbitrary boundaries were used to parcellate the PCR. Relevant boundaries: Inferior: By the RLIC (anteriorly) and the PTR (posteriorly). This was defined arbitrarily by an imaginary horizontal line extending from the midpoint of the $\mathrm{CC}$ splenium in the mid-sagittal plane (Mori et al., 2008). Anterior: The SCR (described previously). Medial: The forceps major and tapetum (TAP; described below) of the CC. Lateral: The superior longitudinal fasciculus (SLF; described below). 


\subsection{3 | Association Fibres}

\section{Cingulum cingular part}

Description: This parcellation defines the frontal component of the cingulum WM within the cingulate gyrus. The cingulate gyrus is located immediately above the $\mathrm{CC}$ and below the cingular sulcus. It curves around the back of the $\mathrm{CC}$ splenium and continues as the hippocampal part of the cingulum $(\mathrm{CGH})$ that enters the mesial temporal lobe (Shah, Jhawar, \& Goel, 2012). Relevant boundaries: Superior: The cingular sulcus. Inferior: The CC. Posterior: This was arbitrarily delineated against the $\mathrm{CGH}$ by an imaginary horizontal line extending from the midpoint of the $\mathrm{CC}$ splenium in the midsagittal plane (Mori et al., 2008). This division corresponds to a change in the dominant fibre orientation identified on the DEC map - from predominantly green-coloured AP-oriented fibres in the CGC to predominantly blue-coloured SI-oriented fibres in the $\mathrm{CGH}$. Lateral: The CC body fibres.

\section{Cingulum Hippocampal Part:}

Description: The CGH courses within the parahippocampal gyrus and terminates anteriorly in the mesial temporal lobe (Shah et al., 2012). Relevant boundaries: Superior: The CGC (described previously). Temporal terminations: We arbitrarily defined this to be at the level of the hippocampal head in the sagittal plane.

\section{Fornix}

Description: This parcellation defines the forniceal crus, body, and column to the level of the anterior commissure (Nieuwenhuys, Voogd, \& Van Hujizen, 2008; Shah et al., 2012). The precommissural column fibres to the septal region were not included due to limited image resolution. The forniceal body can be identified immediately inferiorly to the CC in the mid-sagittal plane. Relevant boundaries: Superior: The body of CC. Anterior and inferior: At the level of the anterior commissure. Posterior: forniceal crus posterior to the thalamus.

\section{Stria terminalis}

Description: This WM tract is the main efferent fibre pathway from the amygdala that courses along the ventricular surface of the thalamus (Nieuwenhuys et al., 2008; Shah et al., 2012). This parcellation also includes the remaining portion of the forniceal crus and fimbria differentiation of these two fibre tracts is not possible due to the current limited image resolution. The forniceal crus courses immediately posterior to the thalamus and medial to the SS (Mori et al., 2008). It continues caudally as the fimbria when entering the mesial temporal lobe (Nieuwenhuys et al., 2008; Shah et al., 2012). Relevant boundaries: Anterior and superior: the forniceal crus (included in the fornix label). Temporal terminations: We defined the fimbria terminations arbitrarily at two axial slices above the level of the $\mathrm{CP}$, at the diencephalon-midbrain junction.

\section{Superior longitudinal fasciculus}

Description: This WM tract provides connections to the frontal, parietal, and temporal lobes (Martino et al., 2013). It is located dorsolaterally to the CR (Mori et al., 2008). Relevant boundaries: Medial: The frontoparietal component of the SLF (contains predominantly green-coloured AP-oriented fibres) is bounded medially by the CR (contains predominantly blue-coloured SI-oriented fibres). The temporal component of the SLF (contains predominantly blue-coloured SI-oriented fibres) is bounded medially by the PTR anteriorly and the SS posteriorly (both of which contain predominantly green-coloured AP-oriented fibres).

\section{External capsule}

Description: This parcellation includes both the EC and extreme capsule, with the intervening GM, the claustrum. Separating these regions is not possible due to limitations in the image resolution. It excludes the portion of the EC containing the inferior fronto-occipital fasciculus (IFO) and the uncinate fasciculus (UFC), both of which are parcellated separately (described below). Relevant boundaries: Superior: The axial slice where the EC and IC merge. Inferior: An arbitrary boundary against the IFO - identified by the changes in the dominant fibre orientation from the DEC map (from predominantly SI-oriented, blue-coloured fibres in the EC to predominantly AP-oriented, greencoloured fibres in the IFO). Medial: The lentiform nucleus and the IC (Mori et al., 2008). Lateral: The insular cortex.

\section{Posterior thalamic radiation}

Description: This parcellation contains WM tracts that connect the caudal thalamus to the occipital and parietal lobes. The fibres are predominantly AP-oriented, green-coloured fibres on the DEC map, and are best visualised on the axial plane. Relevant boundaries: Superior: The PCR (described previously). Inferior: The SS (described below) (Mori et al., 2008). Anterior: The RLIC (described previously).

\section{Sagittal stratum}

Description: This WM region contains long association WM tracts, such as the IFO, optic radiation and PTR, with fibre projections to the occipital lobe. Division against the PTR parcellation is arbitrary. The SS was best visualised on the sagittal plane (Mori et al., 2008). Relevant boundaries: Superior: The RLIC (anteriorly) and the PTR (posteriorly). This superior boundary was arbitrarily defined at the anterior commissure level. Anterior: The IFO (described below) and the UFC (described below).

\section{Superior fronto-occipital fasciculus}

Description: This WM tract, typically vestigial in humans, connects the occipital and frontal lobes and extends posteriorly along the dorsal edge of the caudate nucleus (Forkel et al., 2014; Jellison et al., 2004). Relevant boundaries: Superior and lateral: The SCR (described previously) (Mori et al., 2008). Medial: The head of caudate nucleus and the lateral ventricle.

\section{Inferior fronto-occipital fasciculus}

Description: This WM tract forms the long-ranged frontal and occipital connection (Jellison et al., 2004; Martino, Brogna, Robles, Vergani, \& Duffau, 2010; Mori et al., 2008). This parcellation defines the portion of the IFO that traverses through the EC. Relevant boundaries: Superior: The 
EC. Inferior: The insular segment of the UFC at the temporal stem (Choi, Han, Yee, \& Lee, 2010). Its boundary against the UFC was identified by the changes in the dominant fibre orientation from the DEC map (from predominantly AP-oriented, green-coloured fibres for the IFO to blue-coloured SI-oriented fibres for the UFC) (Ebeling \& von Cramon, 1992; Kier, Staib, Davis, \& Bronen, 2004) Posterior: The SS (described previously) (Jellison et al., 2004). Medial: The lentiform nucleus. Lateral: The insular cortex.

\section{Uncinate fasciculus}

Description: This hook-shaped WM tract connects the orbitofrontal lobe to the anterior temporal lobe via the temporal stem portion of the EC (Ebeling \& von Cramon, 1992; Jellison et al., 2004; Kier et al., 2004). This parcellation defines the insular segment of the UFC that traverses through the temporal stem (Choi et al., 2010). Relevant boundaries: Superior: The IFO (described previously). Inferior: The midbrain-pontine junction. Posterior: The SS (described previously).

\subsection{4 $\quad$ Commissural Fibres}

The CC provides the main inter-hemispheric connections for the cerebrum. We did not parcellate the anterior and posterior commissures due to their small sizes in neonates and the limited image resolution. We adopted Hofer's classification, segmenting the CC into five divisions, based on its AP length at the mid-sagittal plane (Hofer \& Frahm,
2006). The TAP contains the CC temporal fibres and was parcellated as a separate region. The $\mathrm{CC}$ is best located in the mid-sagittal plane, above the lateral ventricles and below the cingulate gyrus. The CR marks the lateral boundaries for the CC parcellations (Mori et al., 2008).

\section{Corpus callosum I}

Description: The $\mathrm{CCl}$ is the anterior $1 / 6$ of the $\mathrm{CC}$ at the mid-sagittal plane. It represents both the rostrum and the genu of the $\mathrm{CC}$ (Hofer \& Frahm, 2006).

\section{Corpus callosum II}

Description: The CCII is defined as the portion of the CC between the anterior $1 / 2$ and the anterior $1 / 6$ of the $C C$ at the mid-sagittal plane. It represents the anterior body of the CC (Hofer \& Frahm, 2006).

\section{Corpus Callosum III}

Description: The CCIII is between the posterior $1 / 2$ and the posterior $1 / 3$ of the $C C$ at the mid-sagittal plane. It represents the posterior body of the CC (Hofer \& Frahm, 2006).

\section{Corpus Callosum IV}

Description: The CCIV is between the posterior $1 / 3$ and the posterior $1 / 5$ of the $C C$ at the mid-sagittal plane. It represents the isthmus of the CC (Hofer \& Frahm, 2006).

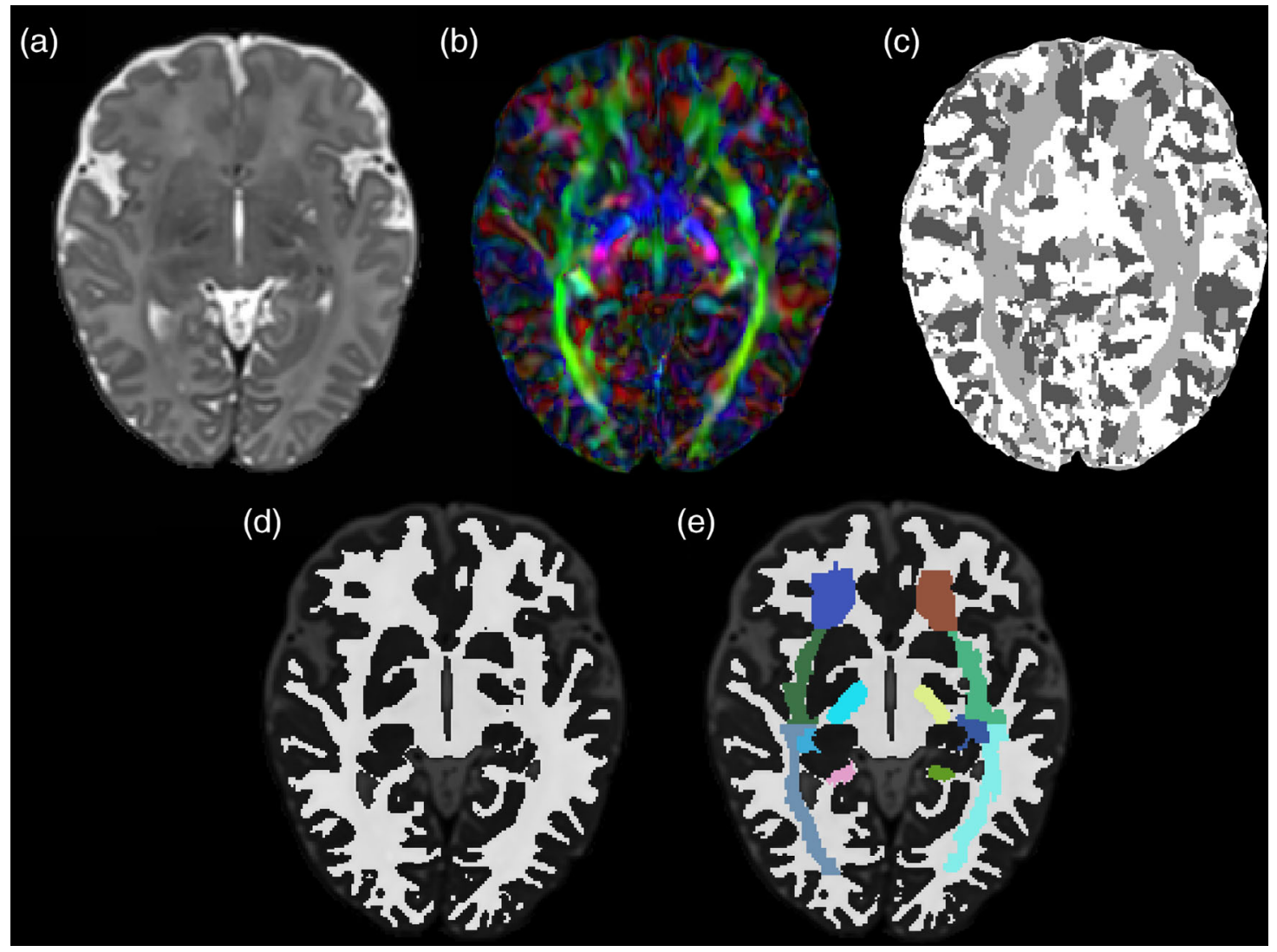

FIGURE 1 Illustration of the stepwise white matter (WM) parcellation process. (a) $T_{2}$-weighted image; (b) Direction encoded colour (DEC) map; (c) Nearest Axis map; (d) WM mask; (e) WM mask with parcellated regions overlaid. Images are displayed in radiological orientation 
TAB LE 1 Complete list of parcellated M-CRIB-WM regions and mean volume for each region

\begin{tabular}{|c|c|c|c|c|c|c|}
\hline Region & & Structure & Predominant direction of travel & Mean volume $\left(\mathrm{mm}^{3}\right)$ & $S D\left(\mathrm{~mm}^{3}\right)$ & Label \\
\hline \multirow[t]{9}{*}{ Tracts in brainstem } & $1^{\mathrm{a}}$ & CST (L) & $\mathrm{SI}$ & 238 & 100 & \\
\hline & 2 & $\mathrm{CST}(\mathrm{R})$ & $\mathrm{SI}$ & 242 & 96 & \\
\hline & 4 & $M L(L)$ & $\mathrm{SI}$ & 316 & 110 & \\
\hline & 5 & $M L(R)$ & $\mathrm{SI}$ & 296 & 110 & \\
\hline & 6 & $\mathrm{SCP}(\mathrm{L})$ & $\mathrm{SI}$ & 99 & 32 & \\
\hline & 8 & $\mathrm{MCP}(\mathrm{L})$ & AP & 682 & 174 & \\
\hline & 9 & $\mathrm{MCP}(\mathrm{R})$ & AP & 696 & 188 & \\
\hline & 10 & $\mathrm{ICP}(\mathrm{L})$ & AP & 74 & 12 & \\
\hline & 11 & $\mathrm{ICP}(\mathrm{R})$ & AP & 75 & 16 & \\
\hline \multirow[t]{11}{*}{ Projection fibres } & 12 & ALIC (L) & AP & 519 & 56 & \\
\hline & 16 & RLIC (L) & $\mathrm{SI}$ & 569 & 82 & \\
\hline & 17 & RLIC (R) & SI & 619 & 117 & \\
\hline & 18 & $\mathrm{ACR}(\mathrm{L})$ & AP & 2,178 & 469 & \\
\hline & 19 & $\mathrm{ACR}(\mathrm{R})$ & AP & 2,293 & 511 & \\
\hline & 20 & $\mathrm{SCR}(\mathrm{L})$ & $\mathrm{SI}$ & 2,956 & 574 & \\
\hline & 21 & $\mathrm{SCR}(\mathrm{R})$ & SI & 2,832 & 603 & \\
\hline & 22 & PCR (L) & SI-AP & 1,157 & 373 & \\
\hline & 23 & PCR (R) & SI-AP & 1,307 & 490 & \\
\hline & 24 & $\mathrm{CP}(\mathrm{L})$ & SI & 300 & 56 & \\
\hline & 25 & $\mathrm{CP}(\mathrm{R})$ & SI & 309 & 59 & \\
\hline \multirow{15}{*}{ Association fibres } & 33 & $\mathrm{ST}(\mathrm{R})$ & LR & 251 & 55 & \\
\hline & 34 & $\operatorname{SLF}(\mathrm{L})$ & $\mathrm{SI}$ & 2,290 & 424 & \\
\hline & 35 & $\operatorname{SLF}(R)$ & SI & 2,408 & 359 & \\
\hline & 36 & $\mathrm{EC}(\mathrm{L})$ & $\mathrm{SI}$ & 1,319 & 206 & \\
\hline & 37 & $\mathrm{EC}(\mathrm{R})$ & SI & 1,291 & 206 & \\
\hline & 38 & PTR (L) & AP & 1,563 & 198 & \\
\hline & 39 & PTR (R) & AP & 1,436 & 184 & \\
\hline & 40 & SS (L) & AP & 976 & 147 & \\
\hline & 41 & SS (R) & AP & 983 & 188 & \\
\hline & 42 & SFO (L) & AP & 148 & 52 & \\
\hline & 43 & SFO (R) & AP & 142 & 56 & \\
\hline & 44 & IFO (L) & AP & 689 & 180 & \\
\hline & 45 & IFO (R) & AP & 706 & 166 & \\
\hline & 46 & UFC (L) & $\mathrm{SI}$ & 159 & 64 & \\
\hline & 47 & UFC (R) & SI & 176 & 64 & \\
\hline
\end{tabular}


TABLE 1 (Continued)

\begin{tabular}{lllllll} 
Region & & Structure & Predominant direction of travel & Mean volume $\left(\mathrm{mm}^{3}\right)$ & SD $\left(\mathrm{mm}^{3}\right)$ & Label \\
Commissural fibres & 48 & CC (I) & LR & 1,109 & 188 \\
& 49 & CC (II) & LR & 948 & 137 \\
& 50 & CC (III) & LR & 346 & 73 \\
& 51 & CC (IV) & LR & 211 & 50 \\
& 52 & CC (V) & LR & 1,333 & 358 \\
& 53 & TAP (L) & SI & 200 & 71 \\
& 54 & TAP (R) & SI & 226 & 79 \\
\hline
\end{tabular}

Note: Labels files containing label indices are provided via the publicly available data set at https://osf.io/mnwv9/

Abbreviations: ACR, anterior corona radiata; AP, anterior-posterior; ALIC, anterior limb of internal capsule; CC, corpus callosum; CGC, cingulum cingular part; CGH, cingulum hippocampal part; CP, cerebral peduncle; EC, external capsule; Fx, Fornix; IFO, inferior fronto-occipital fasciculus; ICP, inferior cerebellar peduncle; LR, left-right; M-CRIB-WM, white matter Melbourne Children's Regional Infant Brain; PCR, posterior corona radiata; PLIC, posterior limb of internal capsule; PTR, posterior thalamic radiation; RLIC, retrolenticular limb of internal capsule; SCR, superior corona radiata; SFO, superior fronto-occipital fasciculus; SI, superior-inferior; ST, stria terminalis; SS, sagittal stratum; SLF, superior longitudinal fasciculus; UFC, uncinate fasciculus; TAP, tapetum.

${ }^{\mathrm{a}}$ Numbers listed are not label indices.

\section{Corpus Callosum V}

Description: The CCV is the posterior $1 / 5$ of the $C C$ at the mid-sagittal plane. It represents the splenium of the CC (Hofer \& Frahm, 2006).

\section{Tapetum}

Description: The TAP is best located within the lateral ventricular wall at the level of ventricular trigone in the occipital lobe. It is medial to the SS and can be readily distinguished from the SS on the DEC map due to differences in the predominant fibre orientation (TAP contains predominantly blue-coloured, SI-oriented temporal CC fibres; the SS contains predominantly green-coloured, AP-oriented association fibres) (Mori et al., 2008).

\section{4 | Manual WM parcellation methods}

All WM structures were delineated manually on the $T_{2}$-weighted images and co-registered DEC maps in volume space using Insight Toolkit (ITK)-SNAP v 3.6 .0 (http://www.nitrc.org/projects/itk-snap/, RRID:SCR_002010) (Yushkevich et al., 2006), which simultaneously displays axial, sagittal, and coronal views along with a composite 3D surface representation of utilised labels.

To aid parcellation of regions containing boundaries between multiple adjacent WM tracts, we constructed individual Nearest Axis maps (see script in Appendix) where each voxel was assigned a value representing the nearest image axis (AP, LR, or IS) to its principal direction of diffusion, based on angular distance. These maps were used to clarify the region boundaries in situations where the primary fibre direction was not obvious via visual inspection of the DEC map. This creates artificial boundaries to help distinguish between voxels containing tracts running, for example, predominantly SI adjacent to voxels containing tracts running AP.

We overlaid the following parameter maps on $T_{2}$-weighted structural images and utilised them in a stepwise manner, in order to identify boundaries and remove boundary ambiguity between different WM regions. First, we overlaid the co-registered DEC map, which revealed the principal fibre direction in each WM region. Many regions comprised some boundaries defined based on anatomical landmarks from the $T_{2}$-weighted images, and some boundaries based on diffusion direction from the DEC maps. Thus, in the first instance, delineating all boundaries of a region involved toggling between the $T_{2}$-weighted image and the DEC map. Next, the co-registered Nearest Axis map was overlaid to aid boundary definition in cases where the DEC-based anatomical boundary was ambiguous. Voxels were only selected if they had the nearest image axis to the principal fibre direction for the WM region, as shown on the Nearest Axis map. Then, the parcellation boundaries were further checked against the underlying $T_{2}$-weighted images to ensure that they conformed with any structural landmarks specified as boundaries.

Manual parcellation was performed and checked on a combination of axial, sagittal and coronal slices, leveraging the clearest perspective available for each WM structure. Parcellation was performed brain-bybrain, rather than region-by-region. This was considered most efficient and effective because boundaries of neighbouring structures are often interdependent, and the visibility of adjacent structures provided much insight in delineating the boundaries of the current parcellated region. In a few regions where anatomy was less clear due to small or subtle structures or partial voluming (e.g., as seen in the fornix), tracing in a single region was then checked in all brains and edited for consistency if needed. The CC was parcellated first in each brain, followed by the cingulum. The IC and EC were then traced. The IC segmentations provided shared boundaries for the PTR, SLF, and TAP.

Although all planes were used to view and check parcellations, some structures were primarily traced in a combination of the sagittal and coronal planes as these offered the clearest contrast/information, including the $\mathrm{CC}$, cingulum, and fornix. Other structures were primarily traced in the axial plane, including the IC and EC, PTR, SLF, TAP, CP, SCP, MCP, ICP, CST, PCT, and ML. The coronal plane was used to 


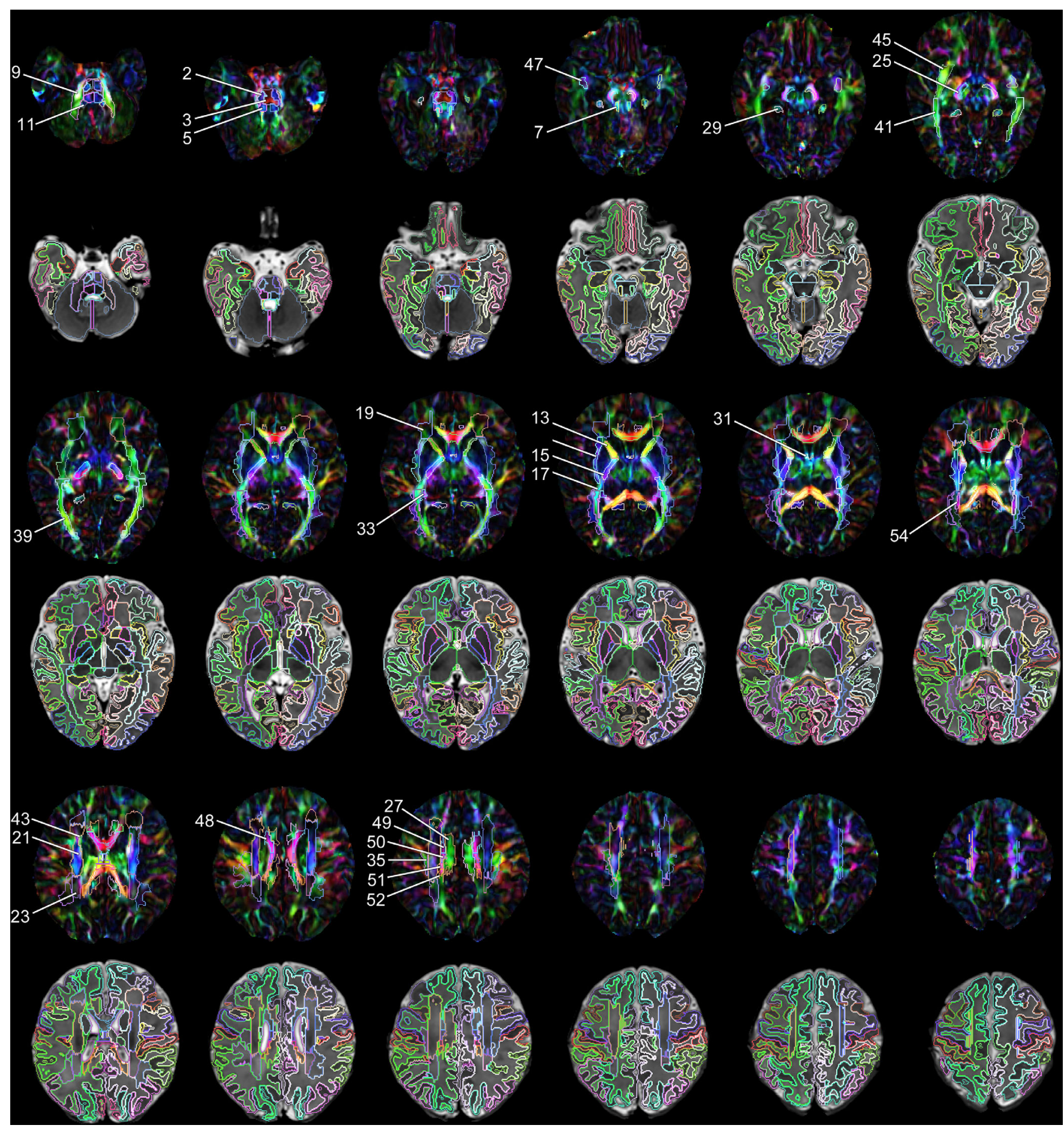

FIGURE 2 Selected axial slices of M-CRIB-WM white matter parcellations (first, third, and fifth rows) overlaid on DEC images; and M-CRIBWM parcellations combined with M-CRIB 2.0 atlas GM regions (second, fourth, and sixth rows) overlaid on $T_{2}$-weighted images. Images are for a single participant, shown in 5-slice increments. Images are displayed in radiological orientation. Annotated region numbers correspond to those listed in Table 1. For full detail, see the online, high-resolution version of this image

cross check the accuracy of regional boundaries. The CP, SCP, MCP and ICP are also intuitive to trace based on the $T_{2}$ images.

Manual parcellation of all 54 WM structures was completed in all 10 individuals by one operator (S.Y.). A neurosurgery research fellow (J.Y., who has over 8 years of diffusion MRI research experience and over 6 years of neurosurgical practice), and a paediatric radiologist
(M.W., who has over 10 years of paediatric radiology practice) confirmed the accuracy of each region's boundaries on all brains, based on the proposed parcellation scheme used in this study. Training for performing segmentations involved studying WM anatomy as pertaining to the JHU neonatal atlas and other literature cited in Section 2.3. When tracing was begun, for the first two brains, tracing 

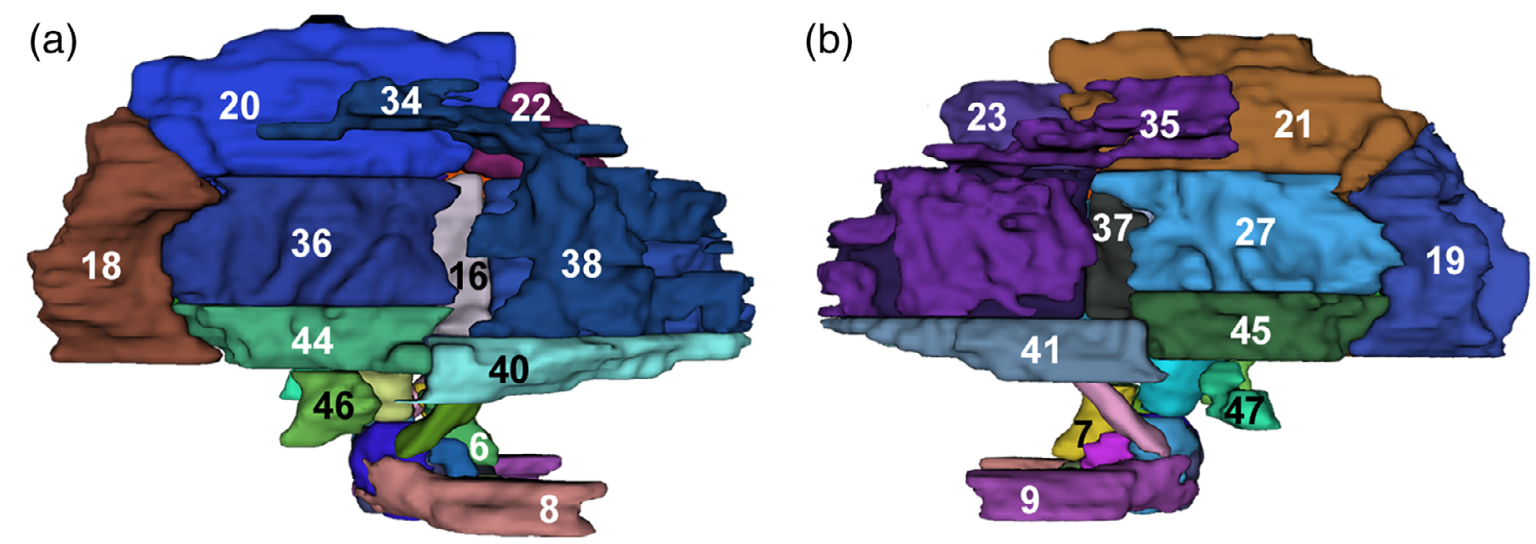

(c)
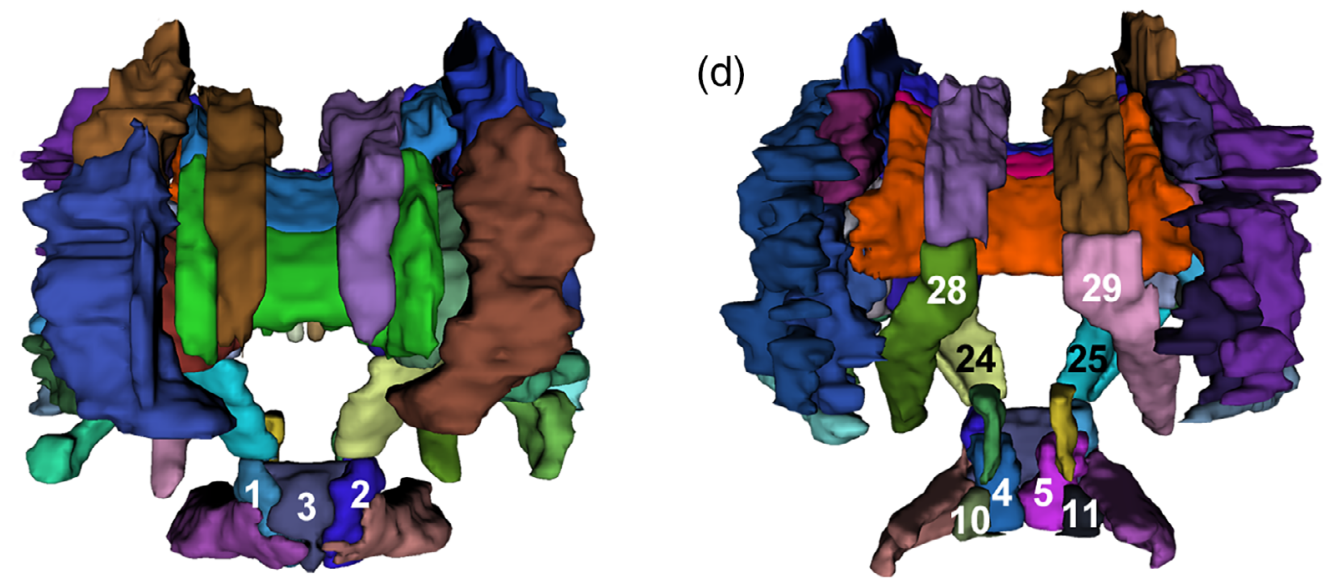

(e)
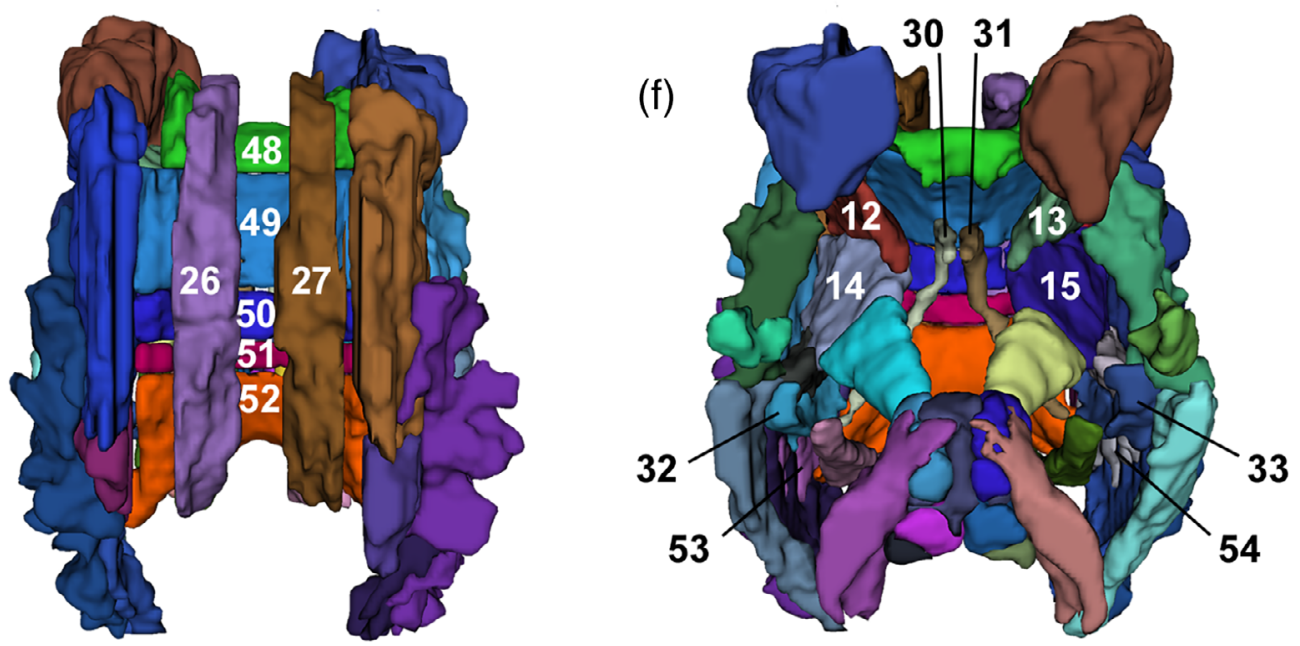

FIGURE 3 Annotated 3D representation of all white matter parcellations for a single participant. (a) Left hemisphere; (b) right hemisphere; (c) frontal view; (d) occipital view; (e) superior view; (f) inferior view. Surfaces underwent Gaussian smoothing with SD 0.8 mm for display purposes. Labels correspond to structures listed in Table 1. Labels 42 and 43 (not shown) correspond to the superior fronto-occipital fasciculi, which are not visible from the angles displayed, however, are shown in Figure 2

was performed and the accuracy of each region was checked and adjusted if needed in consultation with J.Y.

Parcellations for each individual were then masked using the same individual's WM tissue mask. This mask was created by combining and binarising the individual' WM-containing labels from the M-CRIB 2.0 (Alexander et al., 2019) $T_{2}$-based parcellated atlas; specifically, cerebral
WM, CC, ventral diencephalon, brainstem, and cerebellar WM labels (Figure 1). The binarised WM mask was then multiplied by the parcellated WM atlas image using 'fslmaths' tools available as part of the FSL suite (Jenkinson, Beckmann, Behrens, Woolrich, \& Smith, 2012; Smith et al., 2004). This allowed M-CRIB-WM parcellations to be combined with the whole-brain M-CRIB 2.0 atlas parcellations. 


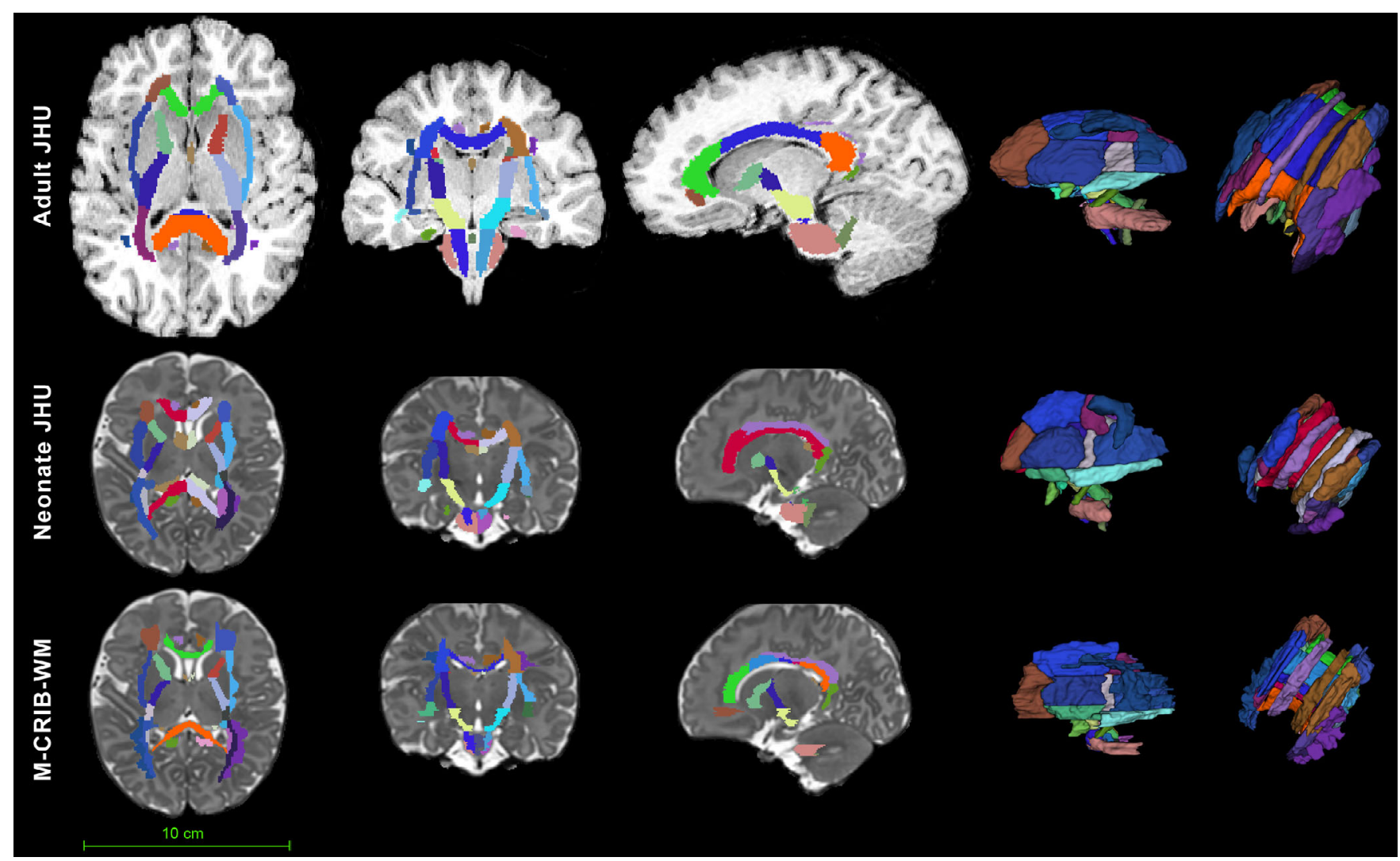

FIGURE 4 Comparison of the adult JHU white matter atlas, JHU-neonate-SS atlas, and the M-CRIB-WM atlas. Top row: $T_{1}$-weighed image of a healthy 18-year-old brain that has been labelled with the adult JHU atlas (Mori \& van Zijl, 2007). Middle row: $T_{2}$-weighed image of a single neonatal participant from the M-CRIB-WM sample, labelled with the JHU-neonate-SS (Oishi et al., 2011) atlas. For clarity of comparison, the labels shown are a subset of the $122 \mathrm{JHU}$-neonate-SS labels, comprising WM regions and tracts for which corresponding structures have been defined in the M-CRIB-WM atlas. Both the neonatal and adult JHU atlas labels were applied by performing nonlinear (symmetric diffeomorphic

normalisation) warping of individual structural images to JHU templates using ANTS, and then applying the inverse transformations and warps to the label images. Bottom row: $T_{2}$-weighed image of the same single neonatal participant from the M-CRIB-WM sample, with manually parcellated labels overlaid. Relative size of the adult and neonate brains is to scale

Manual parcellation of the 10 brains occurred part-time over a period of 12 months. Training for WM regional boundary definition took approximately 160 hours. Manual label tracing took approximately 25 hours per brain. Reviewing all brain parcellations with an expert took approximately 20 hours, and subsequent editing to refine the parcellations from the 10 brains occupied approximately 30 hours in total.

\section{3}

\section{RESULTS}

The WM extension of the M-CRIB atlases comprises 24 pairs of leftand right-hemispheric structures, and six single structures, totalling 54 regions. A full list of parcellated WM regions along with their mean volume and standard deviation is included in Table 1. Combining the M-CRIB-WM WM regions with the original M-CRIB (Alexander et al., 2017) whole-brain atlas, results in a parcellated atlas comprising 154 regions altogether. Combining the WM regions with the M-CRIB 2.0 (Alexander et al., 2019) whole brain atlas results in 148 parcellated regions. Selected axial slices of the WM parcellations and combined
M-CRIB 2.0 and WM parcellations for a single participant are illustrated in Figure 2. Figure 3 depicts a surface representation of all WM parcellations for a single participant. Figure 4 illustrates volumetric and surface-based M-CRIB-WM parcellations for a single neonatal participant compared with equivalent JHU-neonate-SS (Oishi et al., 2011) WM labels, and compared with an adult $T_{1}$-weighted brain image labelled using the equivalent adult $\mathrm{JHU}$ parcellation scheme (Mori et al., 2008), to illustrate compatibility of the parcellated regions between these time points.

Figure S1 presents surface representations of all 10 parcellated WM atlases. Figure S2 presents surface representations of combined WM and cortical regions in a single participant. Figure S3 presents selected axial slices of all 10 individual parcellated WM atlases.

The M-CRIB-WM individual atlas data sets are publicly available via https://osf.io/mnwv9/, or linked to via https://github.com/ DevelopmentallmagingMCRI. The data include parcellated, $T_{2^{-}}$ weighed, $T_{1}$-weighed, and DEC NiFTI images for each individual, along with labels files (with corresponding label numbers and descriptions), and basic participant data (sex, age at birth, age at scan) are provided as text files. Individual datasets for a combined atlas comprising both 
M-CRIB 2.0 regions (basal ganglia, thalamus, cerebellum, cortex, and other regions) and the current WM regions is also provided. The data are available under a 'CC-By Attribution 4.0 International' creative commons licence, which permits reuse of the data with attribution.

\section{4 | DISCUSSION}

In this work, we present the WM extension to our existing M-CRIB atlases, the M-CRIB-WM. This atlas contains 54 manually parcellated WM regions, in 10 healthy term neonates. The M-CRIB-WM has been defined based on high-quality neonatal DWI and $T_{2}$-weighted data, enabling delineation of the relatively small, detailed structures in the neonatal brain. The use of manual segmentation allowed us to precisely segment structures in each individual.

Manual segmentation remains the best practice for MRI brain parcellation as it allows precise delineation of different brain regions, particularly those with complex or arbitrarily defined boundaries. The WM of the brain, comprising a complex network of neuronal axons, typically has indistinct boundaries between neighbouring WM tracts. For example, the AP-oriented fibres of the IFO, optic radiation, and PTR traverse through the SS, and cannot be differentiated macroscopically even with meticulous cadaveric fibre dissection techniques (Yasargil, Ture, \& Yasargil, 2004). In other instances, the only discernible feature between neighbouring WM tracts is the difference in the dominant fibre orientation. For example, the IFO and UFC are differentiable in the temporal stem because one (IFO) has AP-oriented fibres, forming the fronto-occipital connections, whereas the other (UFC) has SI-oriented fibres and hooks around the temporal stem, forming the frontotemporal connections (Choi et al., 2010; Ebeling \& von Cramon, 1992; Kier et al., 2004). The WM regions are defined for convenience in anatomical studies. The distinction between neighbouring WM regions is also largely arbitrary. While some WM regions can be defined structurally based on anatomical landmarks (e.g., the CP), there are other WM regions where arbitrary boundaries are unavoidable. In particular, the deep WM regions (ACR, SCR, and PCR) lack clear, recognisable anatomical boundaries between the neighbouring regions. Here, we used imaginary lines drawn perpendicularly from the back edge of the genu of the $\mathrm{CC}$, and the front edge of the splenium of the $\mathrm{CC}$ to divide the three portions of the $\mathrm{CR}$, a technique we developed through visual inspection of the JHU adult brain atlas. Although arbitrary, we found this boundary definition reproducible through all subjects.

Terminology that is anatomically clear can reduce ambiguity and bias and allow consistency across different operators. We based our region definitions on those provided for the JHU-neonate-SS atlas and have further elaborated boundary definitions for the purpose of clarity. For example, the CST label consists of only the ventral pontine portion of this WM tract in the $\mathrm{JHU}$ atlases, rather than the entire tract. The resulting detailed parcellation protocols that we have provided for all $54 \mathrm{WM}$ regions, as an elaboration of those provided for the JHU atlases, are a strength of the M-CRIB-WM atlas.

A challenge when performing manual parcellation based on DWI is that the dominant colour intensities on the DEC map may be ambiguous, particularly at regions where multiple WM tracts intersect. Our approach to this problem was to develop a discretised Nearest Axis map that indexes only the principal direction (i.e., AP, SI, or LR) in each voxel. This facilitated the identification of a predominant colour on the corresponding DEC map, and thus clarified the principal fibre direction at the boundary between neighbouring regions. This provided a decision solution that enabled us to define anatomical regions with an increased level of certainty. Another strength is that all 10 brains were delineated by a single operator, thus eliminating inter-rater bias. Although individual bias is a possibility, we were careful to receive detailed feedback on the label boundaries of each brain by both an independent neurosurgery fellow and a paediatric radiologist.

We describe our parcellated WM regions as compatible with the $\mathrm{JHU}$-neonate-SS regions and adult JHU white matter atlas regions, with the implication that properties of the current regions may be compared with those at later time points labelled with the adult atlas. In doing so, we acknowledge that the boundary definitions have been adapted and further defined here, and that the regions were traced by different operators to those who developed the JHU atlases. As such, there may be some differences in region boundaries or their interpretations by the operators, and parcellation differences based on the resolution, sample participants, and sample size of the data. As described in Section 2, there are also some regions where parcellations differed from those in the JHU-neonate-SS atlas, and it is necessary to take these into account when designing studies. Users may choose to accept these caveats in view of the advantages this dataset offers.

Considering the time-consuming nature of the manual segmentation process, the M-CRIB-WM atlas is a valuable and unparalleled resource. Whereas other existing detailed parcellated neonatal atlases have been defined in a single subject and/or propagated from other developmental time points, the current atlas comprises 10 individually manually parcellated neonatal brains. A training set of this size has been observed in adult data to be sufficient to represent individual variability in morphology (Croxson et al., 2018; Heckemann et al., 2006), and although there do not appear to be equivalent studies at the neonatal time point, this may at least be a reasonable indication of what may be minimally sufficient in neonates. Parcellation tools can then draw upon labelled multi-subject training sets by applying multiatlas label fusion or emerging deep learning algorithms, which leverage this variability in different ways to optimise labelling accuracy (see reviews by, e.g., Lin \& Li, 2019; Makropoulos, Counsell, \& Rueckert, 2018). There are multiple available segmentation and parcellation tools for neonatal data that contain pre-packaged atlases or tissue priors (e.g., Beare et al., 2016; Makropoulos et al., 2018; Makropoulos, Counsell, \& Rueckert, 2018; Otsuka et al., 2019; Zollei, Ou, Iglesias, Grant, \& Fischl, 2017), although these did not incorporate the MCRIB-WM at the time of publication. To use the current training set to perform parcellation, examples of available tools that allow users to input external volumetric parcellated training sets such as the current atlas include ANTS (Avants et al., 2011), and STAPLE or pSTAPLE (Akhondi-Asl \& Warfield, 2013; Warfield, Zou, \& Wells, 2004). These tools rely on diffeomorphic registration and use label fusion 
algorithms to parcellate data. Future work with the current data may assess the benefits of incorporating DWI in the registration of training and test data, together with $T_{2}$-weighted images. The public release of the current dataset means that it is available for future incorporation as an atlas into existing parcellation tools and may also be used as ground truth for methodological work.

Considering there are equivalent $\mathrm{JHU}$ atlases available for younger third trimester time points (Feng et al., 2019) and older time points (Mori et al., 2008; Oishi et al., 2009), our neonatal WM atlas also has the benefit of facilitating longitudinal analyses of neuroimaging metrics from equivalent WM regions. Together with the large range of complementary multi-parametric neuroimaging tools and techniques available, the combined M-CRIB and M-CRIB-WM atlases will enable detailed structural and microstructural measures to be obtained in an accurate and age-specific way for major cortical and subcortical regions of the neonatal brain, and now additionally for all major WM tracts and regions. To our knowledge, there has not previously existed a single neonatal atlas encompassing the parcellation of both extensive GM and WM regions to a satisfactory level of detail. Our novel M-CRIB-WM atlas, along with the M-CRIB cortical and subcortical atlases, provides neonatal whole brain MRI coverage of standardised GM and WM parcellations. This addition will greatly benefit the field of infant neuroimaging research.

In summary, we have presented a neonatal WM atlas capturing important WM structural variability unique to, and characteristic of, the neonatal time point. The individual parcellated image maps and structural templates of the M-CRIB-WM neonatal atlas, including a complete atlas comprising both M-CRIB 2.0 regions (basal ganglia, thalamus, cerebellum, cortex, and other regions) and the current WM regions, are publicly available at https://osf.io/mnwv9/ or via https://github.com/ DevelopmentallmagingMCRI. This novel atlas will provide extensive neonatal brain coverage with substantial anatomic detail. It will be a valuable resource that will help facilitate investigation of brain structure at the neonatal time point and developmentally across the lifespan.

\section{ACKNOWLEDGMENTS}

The authors gratefully acknowledge ideas and support provided by the members of the Victorian Infant Brain Study (VIBeS) and Developmental Imaging groups, as well as the Melbourne Children's MRI Centre, located at the Murdoch Children's Research Institute, Melbourne, Victoria. The authors also thank the families who participated in this study. This project was supported by the Australian National Health and Medical Research Council (NHMRC) (Project Grant ID 1028822 and 1024516; Centre of Clinical Research Excellence Grant ID 546519; Centre of Research Excellence Grant ID 1060733 and 1153176; Senior Research Fellowship ID 1081288 to P.J.A.; Early Career Fellowship ID 1053787 to J.L.Y.C., ID 1053767 to A.J.S., ID 1012236 to D.K.T.; Career Development Fellowship ID 1108714 to A.J.S., ID 1085754 and 1160003 to D.K.T.), the Royal Children' Hospital Foundation (RCH 1000 to J.Y.M.Y.), Brigham and Women' Program for Interdisciplinary Neuroscience Travelling Neuroscience Fellowship (to L.G.M), Murdoch Children' Research Institute, The University of Melbourne Department of Paediatrics, and the Victorian
Government's Operational Infrastructure Support Program. The funding sources had no involvement in the study design; in the collection, analysis and interpretation of data; in the writing of the report; and in the decision to submit the article for publication.

\section{CONFLICT OF INTEREST}

The authors declare no potential conflict of interest.

\section{DATA AVAILABILITY STATEMENT}

Data availability statement: The data that support the findings of this study are openly available at https://osf.io/mnwv9/ or via https:// github.com/DevelopmentallmagingMCRI.

\section{ORCID}

Bonnie Alexander (iD) https://orcid.org/0000-0003-1108-5890 Joseph Yuan-Mou Yang (D) https://orcid.org/0000-0003-4081-7157 Claire E. Kelly (D) https://orcid.org/0000-0002-6564-0872 Gareth Ball (D) https://orcid.org/0000-0003-3509-1435 Deanne K. Thompson (DD https://orcid.org/0000-0001-8017-5756

\section{REFERENCES}

Akhondi-Asl, A., Hoyte, L., Lockhart, M. E., \& Warfield, S. K. (2014). A logarithmic opinion pool based STAPLE algorithm for the fusion of segmentations with associated reliability weights. IEEE Transactions on Medical Imaging, 33(10), 1997-2009. https://doi.org/10.1109/TMI. 2014.2329603

Akhondi-Asl, A., \& Warfield, S. K. (2013). Simultaneous truth and performance level estimation through fusion of probabilistic segmentations. IEEE Transactions on Medical Imaging, 32(10), 1840-1852.

Alexander, B., Kelly, C. E., Adamson, C., Beare, R., Zannino, D., Chen, J., ... Thompson, D. K. (2019). Changes in neonatal regional brain volume associated with preterm birth and perinatal factors. Neurolmage, 185, 654-663. https://doi.org/10.1016/j.neuroimage.2018.07.021

Alexander, B., Murray, A. L., Loh, W. Y., Matthews, L. G., Adamson, C., Beare, R., ... Thompson, D. K. (2017). A new neonatal cortical and subcortical brain atlas: The Melbourne Children's regional infant brain (MCRIB) atlas. Neurolmage, 147, 841-851. https://doi.org/10.1016/j. neuroimage.2016.09.068

Anderson, P. J., Cheong, J. L., \& Thompson, D. K. (2015). The predictive validity of neonatal MRI for neurodevelopmental outcome in very preterm children. Seminars in Perinatology, 39(2), 147-158 WB Saunders.

Avants, B. B., Epstein, C. L., Grossman, M., \& Gee, J. C. (2008). Symmetric diffeomorphic image registration with cross-correlation: Evaluating automated labeling of elderly and neurodegenerative brain. Medical Image Analysis, 12(1), 26-41. https://doi.org/10.1016/j.media.2007. 06.004

Avants, B. B., Tustison, N. J., Song, G., Cook, P. A., Klein, A., \& Gee, J. C. (2011). A reproducible evaluation of ANTs similarity metric performance in brain image registration. Neurolmage, 54(3), 2033-2044. https://doi.org/10.1016/j.neuroimage.2010.09.025

Beare, R., Chen, J., Alexopoulos, D., Kelly, C. E., Smyser, C., Rogers, C., ... Thompson, D. K. (2016). Neonatal brain tissue classification with morphological adaptation and unified segmentation. Frontiers in $\mathrm{Neu}$ roinformatics, 10, 12. https://doi.org/10.3389/fninf.2016.00012

Blesa, M., Serag, A., Wilkinson, A. G., Anblagan, D., Telford, E. J., Pataky, R., ... Boardman, J. P. (2016). Parcellation of the healthy neonatal brain into 107 regions using atlas propagation through intermediate time points in childhood. Frontiers in Neuroscience, 10, 220. https:// doi.org/10.3389/fnins.2016.00220 
Chang, L., Oishi, K., Skranes, J., Buchthal, S., Cunningham, E., Yamakawa, R., ... Cloak, C. (2016). Sex-specific alterations of white matter developmental trajectories in infants with prenatal exposure to methamphetamine and tobacco. JAMA Psychiatry, 73(12), 1217-1227.

Choi, C. Y., Han, S. R., Yee, G. T., \& Lee, C. H. (2010). An understanding of the temporal stem. Journal of Korean Neurosurgical Association, 47(5), 365-369. https://doi.org/10.3340/jkns.2010.47.5.365

Croxson, P. L., Forkel, S. J., Cerliani, L., \& Thiebaut de Schotten, M. (2018). Structural variability across the primate brain: A cross-species comparison. Cerebral Cortex, 28(11), 3829-3841.

de Macedo Rodrigues, K., Ben-Avi, E., Sliva, D. D., Choe, M. S., Drottar, M., Wang, R., ... Zollei, L. (2015). A FreeSurfer-compliant consistent manual segmentation of infant brains spanning the 0-2 year age range. Frontiers in Human Neuroscience, 9, 21. https://doi.org/10.3389/ fnhum.2015.00021

Desikan, R. S., Segonne, F., Fischl, B., Quinn, B. T., Dickerson, B. C., Blacker, D., ... Killiany, R. J. (2006). An automated labeling system for subdividing the human cerebral cortex on MRI scans into gyral based regions of interest. Neurolmage, 31(3), 968-980. https://doi.org/10. 1016/j.neuroimage.2006.01.021

Dickie, D. A., Shenkin, S. D., Anblagan, D., Lee, J., Blesa Cabez, M., Rodriguez, D., ... Wardlaw, J. M. (2017). Whole brain magnetic resonance image atlases: A systematic review of existing atlases and caveats for use in population imaging. Frontiers in Neuroinformatics, 11, 1. https://doi.org/10.3389/fninf.2017.00001

Dubois, J., Dehaene-Lambertz, G., Kulikova, S., Poupon, C., Huppi, P. S., \& Hertz-Pannier, L. (2014). The early development of brain white matter: A review of imaging studies in fetuses, newborns and infants. Neuroscience, 276, 48-71. https://doi.org/10.1016/j.neuroscience.2013. 12.044

Ebeling, U., \& von Cramon, D. (1992). Topography of the uncinate fascicle and adjacent temporal fiber tracts. Acta Neurochirurgica, 115(3-4), 143-148.

Feng, L., Li, H., Oishi, K., Mishra, V., Song, L., Peng, Q., ... Lee, L. (2019). Age-specific gray and white matter DTI atlas for human brain at 33, 36 and 39 postmenstrual weeks. Neurolmage, 185, 685-698.

Fillmore, P. T., Richards, J. E., Phillips-Meek, M. C., Cryer, A., \& Stevens, M. (2015). Stereotaxic magnetic resonance imaging brain atlases for infants from 3 to 12 months. Developmental Neuroscience, 37(6), 515-532. https://doi.org/10.1159/000438749

Forkel, S. J., Thiebaut de Schotten, M., Kawadler, J. M., Dell'Acqua, F., Danek, A., \& Catani, M. (2014). The anatomy of fronto-occipital connections from early blunt dissections to contemporary tractography. Cortex, 56, 73-84. https://doi.org/10.1016/j.cortex. 2012.09.005

Gousias, I. S., Edwards, A. D., Rutherford, M. A., Counsell, S. J., Hajnal, J. V., Rueckert, D., \& Hammers, A. (2012). Magnetic resonance imaging of the newborn brain: Manual segmentation of labelled atlases in term-born and preterm infants. Neurolmage, 62(3), 1499-1509. https://doi.org/10.1016/j.neuroimage.2012.05.083

Greve, D. N., \& Fischl, B. (2009). Accurate and robust brain image alignment using boundary-based registration. Neurolmage, 48(1), 63-72. https://doi.org/10.1016/j.neuroimage.2009.06.060

Heckemann, R. A., Hajnal, J. V., Aljabar, P., Rueckert, D., \& Hammers, A. (2006). Automatic anatomical brain MRI segmentation combining label propagation and decision fusion. Neurolmage, 33(1), 115-126. https:// doi.org/10.1016/j.neuroimage.2006.05.061

Heemskerk, A. M., Leemans, A., Plaisier, A., Pieterman, K., Lequin, M. H., \& Dudink, J. (2013). Acquisition guidelines and quality assessment tools for analyzing neonatal diffusion tensor MRI data. AJNR American Journal of Neuroradiology, 34(8), 1496-1505. https://doi.org/10.3174/ajnr. A3465

Hirsch, W. L., Kemp, S. S., Martinez, A. J., Curtin, H., Latchaw, R. E., \& Wolf, G. (1989). Anatomy of the brainstem: Correlation of in vitro MR images with histologic sections. AJNR American Journal of Neuroradiology, 10(5), 923-928.

Hofer, S., \& Frahm, J. (2006). Topography of the human corpus callosum revisited-comprehensive fiber tractography using diffusion tensor magnetic resonance imaging. Neurolmage, 32(3), 989-994. https://doi. org/10.1016/j.neuroimage.2006.05.044

Jellison, B. J., Field, A. S., Medow, J., Lazar, M., Salamat, M. S., \& Alexander, A. L. (2004). Diffusion tensor imaging of cerebral white matter: A pictorial review of physics, fiber tract anatomy, and tumor imaging patterns. AJNR American Journal of Neuroradiology, 25(3), 356-369.

Jenkinson, M., Bannister, P., Brady, M., \& Smith, S. (2002). Improved optimization for the robust and accurate linear registration and motion correction of brain images. Neurolmage, 17(2), 825-841.

Jenkinson, M., Beckmann, C. F., Behrens, T. E., Woolrich, M. W., \& Smith, S. M. (2012). FSL. Neurolmage, 62(2), 782-790.

Jenkinson, M., \& Smith, S. (2001). A global optimisation method for robust affine registration of brain images. Medical Image Analysis, 5(2), 143-156.

Kazemi, K., Moghaddam, H. A., Grebe, R., Gondry-Jouet, C., \& Wallois, F. (2007). A neonatal atlas template for spatial normalization of wholebrain magnetic resonance images of newborns: Preliminary results. Neurolmage, 37(2), 463-473. https://doi.org/10.1016/j.neuroimage. 2007.05.004

Kier, E. L., Staib, L. H., Davis, L. M., \& Bronen, R. A. (2004). MR imaging of the temporal stem: Anatomic dissection tractography of the uncinate fasciculus, inferior occipitofrontal fasciculus, and Meyer's loop of the optic radiation. AJNR American Journal of Neuroradiology, 25(5), 677-691.

Kuklisova-Murgasova, M., Aljabar, P., Srinivasan, L., Counsell, S. J., Doria, V., Serag, A., ... Rueckert, D. (2011). A dynamic 4D probabilistic atlas of the developing brain. Neurolmage, 54(4), 2750-2763. https:// doi.org/10.1016/j.neuroimage.2010.10.019

Leemans, A., \& Jones, D. K. (2009). The B-matrix must be rotated when correcting for subject motion in DTI data. Magnetic Resonance in Medicine, 61(6), 1336-1349. https://doi.org/10.1002/mrm.21890

Lin, X., \& Li, X. (2019). Image based brain segmentation: From multi-atlas fusion to deep learning. Current Medical Imaging Reviews, 15(5), 443-452.

Loh, W. Y., Connelly, A., Cheong, J. L., Spittle, A. J., Chen, J., Adamson, C., ... Doyle, L. W. (2016). A new MRI-based pediatric subcortical segmentation technique (PSST). Neuroinformatics, 14(1), 69-81.

Makropoulos, A., Aljabar, P., Wright, R., Huning, B., Merchant, N., Arichi, T., ... Rueckert, D. (2016). Regional growth and atlasing of the developing human brain. Neurolmage, 125, 456-478. https://doi.org/ 10.1016/j.neuroimage.2015.10.047

Makropoulos, A., Counsell, S. J., \& Rueckert, D. (2018). A review on automatic fetal and neonatal brain MRI segmentation. Neurolmage, 170, 231-248.

Makropoulos, A., Robinson, E. C., Schuh, A., Wright, R., Fitzgibbon, S., Bozek, J., ... Passerat-Palmbach, J. (2018). The developing human connectome project: A minimal processing pipeline for neonatal cortical surface reconstruction. Neurolmage, 173, 88-112.

Martino, J., Brogna, C., Robles, S. G., Vergani, F., \& Duffau, H. (2010). Anatomic dissection of the inferior fronto-occipital fasciculus revisited in the lights of brain stimulation data. Cortex, 46(5), 691-699. https:// doi.org/10.1016/j.cortex.2009.07.015

Martino, J., De Witt Hamer, P. C., Berger, M. S., Lawton, M. T., Arnold, C. M., de Lucas, E. M., \& Duffau, H. (2013). Analysis of the subcomponents and cortical terminations of the perisylvian superior longitudinal fasciculus: A fiber dissection and DTI tractography study. Brain Structure \& Function, 218(1), 105-121. https://doi.org/10.1007/ s00429-012-0386-5

Mori, S., Oishi, K., \& Faria, A. V. (2009). White matter atlases based on diffusion tensor imaging. Current Opinion in Neurology, 22(4), 362. 
Mori, S., Oishi, K., Jiang, H., Jiang, L., Li, X., Akhter, K., ... Mazziotta, J. (2008). Stereotaxic white matter atlas based on diffusion tensor imaging in an ICBM template. Neurolmage, 40(2), 570-582. https://doi.org/ 10.1016/j.neuroimage.2007.12.035

Mori, S., \& van Zijl, P. (2007). Human white matter atlas. The American Journal of Psychiatry, 164(7), 1005. https://doi.org/10.1176/ajp.2007. 164.7.1005

Mori, S., W., S., Nagae-Poetscher, L. M., \& van Zijl, P. C. (2005). MRI atlas of human white matter. Amsterdam, The Netherlands: Elsevier.

Nieuwenhuys, R., Voogd, J., \& Van Hujizen, C. (2008). The human central nervous system (4th ed.). Heidelberg/Berlin: Steinkopff-Verlag.

Oishi, K., Faria, A., Jiang, H., Li, X., Akhter, K., Zhang, J., ... Mori, S. (2009). Atlas-based whole brain white matter analysis using large deformation diffeomorphic metric mapping: Application to normal elderly and Alzheimer's disease participants. Neurolmage, 46(2), 486-499.

Oishi, K., Mori, S., Donohue, P. K., Ernst, T., Anderson, L., Buchthal, S., ... Chang, L. (2011). Multi-contrast human neonatal brain atlas: Application to normal neonate development analysis. Neurolmage, 56(1), 8-20. https://doi.org/10.1016/j.neuroimage.2011.01.051

Otsuka, Y., Chang, L., Kawasaki, Y., Wu, D., Ceritoglu, C., Oishi, K., ... Oishi, K. (2019). A multi-atlas label fusion tool for neonatal brain MRI Parcellation and quantification. Journal of Neuroimaging, 29(4), 431-439.

Pannek, K., Hatzigeorgiou, X., Colditz, P. B., \& Rose, S. (2013). Assessment of structural connectivity in the preterm brain at term equivalent age using diffusion MRI and T2 relaxometry: A network-based analysis. PLoS One, 8(8), e68593.

Richards, J. E., Sanchez, C., Phillips-Meek, M., \& Xie, W. (2016). A database of age-appropriate average MRI templates. Neurolmage, 124(Pt B), 1254-1259. https://doi.org/10.1016/j.neuroimage.2015.04.055

Sanchez, C. E., Richards, J. E., \& Almli, C. R. (2012). Neurodevelopmental MRI brain templates for children from 2 weeks to 4 years of age. Developmental Psychobiology, 54(1), 77-91. https://doi.org/10.1002/ dev.20579

Shah, A., Jhawar, S. S., \& Goel, A. (2012). Analysis of the anatomy of the Papez circuit and adjoining limbic system by fiber dissection techniques. Journal of Clinical Neuroscience, 19(2), 289-298. https://doi. org/10.1016/j.jocn.2011.04.039

Shi, F., Yap, P. T., Fan, Y., Gilmore, J. H., Lin, W., \& Shen, D. (2010). Construction of multi-region-multi-reference atlases for neonatal brain MRI segmentation. Neurolmage, 51(2), 684-693. https://doi.org/10. 1016/j.neuroimage.2010.02.025

Shi, F., Yap, P. T., Wu, G., Jia, H., Gilmore, J. H., Lin, W., \& Shen, D. (2011). Infant brain atlases from neonates to 1- and 2-year-olds. PLoS One, 6 (4), e18746. https://doi.org/10.1371/journal.pone.0018746

Smith, S. M. (2002). Fast robust automated brain extraction. Human Brain Mapping, 17(3), 143-155.

Smith, S. M., Jenkinson, M., Woolrich, M. W., Beckmann, C. F., Behrens, T. E., Johansen-Berg, H., ... Flitney, D. E. (2004). Advances in functional and structural MR image analysis and implementation as FSL. Neurolmage, 23, S208-S219. https://doi.org/10.1016/j. neuroimage.2004.07.051

Spittle, A. J., Thompson, D. K., Brown, N. C., Treyvaud, K., Cheong, J. L., Lee, K. J., ... Anderson, P. J. (2014). Neurobehaviour between birth and 40 weeks' gestation in infants born $<30$ weeks' gestation and parental psychological wellbeing: Predictors of brain development and child outcomes. BMC Pediatrics, 14, 111. https://doi.org/10.1186/14712431-14-111

Thompson, D. K., Inder, T. E., Faggian, N., Johnston, L., Warfield, S. K., Anderson, P. J., ... Egan, G. F. (2011). Characterization of the corpus callosum in very preterm and full-term infants utilizing MRI. Neurolmage, 55(2), 479-490. https://doi.org/10.1016/j.neuroimage. 2010.12.025

Thompson, D. K., Inder, T. E., Faggian, N., Warfield, S. K., Anderson, P. J., Doyle, L. W., \& Egan, G. F. (2012). Corpus callosum alterations in very preterm infants: Perinatal correlates and 2 year neurodevelopmental outcomes. Neurolmage, 59(4), 3571-3581.

Thompson, D. K., Kelly, C. E., Chen, J., Beare, R., Alexander, B., Seal, M. L., ... Spittle, A. J. (2018). Characterisation of brain volume and microstructure at term-equivalent age in infants born across the gestational age spectrum. Neuroimage Clinical, 21, 101630. https://doi.org/10. 1016/j.nicl.2018.101630

Ture, U., Yasargil, M. G., Friedman, A. H., \& Al-Mefty, O. (2000). Fiber dissection technique: Lateral aspect of the brain. Neurosurgery, 47(2), 417-426 discussion 426-417.

Tustison, N. J., Avants, B. B., Cook, P. A., Zheng, Y., Egan, A., Yushkevich, P. A., \& Gee, J. C. (2010). N4ITK: Improved N3 bias correction. IEEE Transactions on Medical Imaging, 29(6), 1310-1320. https://doi.org/10.1109/TMI.2010.2046908

Tzourio-Mazoyer, N., Landeau, B., Papathanassiou, D., Crivello, F., Etard, O., Delcroix, N., ... Joliot, M. (2002). Automated anatomical labeling of activations in SPM using a macroscopic anatomical parcellation of the MNI MRI single-subject brain. Neurolmage, 15(1), 273-289. https://doi.org/10.1006/nimg.2001.0978

Walsh, J. M., Doyle, L. W., Anderson, P. J., Lee, K. J., \& Cheong, J. L. (2014). Moderate and late preterm birth: Effect on brain size and maturation at term-equivalent age. Radiology, 273(1), 232-240. https:// doi.org/10.1148/radiol.14132410

Warfield, S. K., Zou, K. H., \& Wells, W. M. (2004). Simultaneous truth and performance level estimation (STAPLE): An algorithm for the validation of image segmentation. IEEE Transactions on Medical Imaging, 23(7), 903-921. https://doi.org/10.1109/TMI.2004. 828354

Yasargil, M. G., Ture, U., \& Yasargil, D. C. (2004). Impact of temporal lobe surgery. Journal of Neurosurgery, 101(5), 725-738. https://doi.org/10. 3171/jns.2004.101.5.0725

Yushkevich, P. A., Piven, J., Hazlett, H. C., Smith, R. G., Ho, S., Gee, J. C., \& Gerig, G. (2006). User-guided 3D active contour segmentation of anatomical structures: Significantly improved efficiency and reliability. Neurolmage, 31(3), 1116-1128. https://doi.org/10.1016/j.neuro image.2006.01.015

Zollei, L., Ou, Y., Iglesias, J., Grant, P. E., \& Fischl, B. (2017). FreeSurfer image processing pipeline for infant clinical MRI images. Paper presented at the Human Brain Mapping, Vancouver, Canada.

\section{SUPPORTING INFORMATION}

Additional supporting information may be found online in the Supporting Information section at the end of this article.

How to cite this article: Alexander B, Yang JY-M, Yao SHW, et al. White matter extension of the Melbourne Children's Regional Infant Brain atlas: M-CRIB-WM. Hum Brain Mapp. 2020;41:2317-2333. https://doi.org/10.1002/hbm.24948 


\section{APPENDIX A.}

Script for co-registered Nearest Axis map used to aid in boundary delineation.

\#! /bin/bash

\# diffusion_directions.sh

\# creates a 3-class image with classes defined by

closest axis to the principal direction of diffusion in each voxel

\# usage:

\#./diffusion_directions.sh

\# eg: ./diffusion_directions.shimg_v1.nii.gz

img_mask.nii.gz

\# output: xyz.nii.gz

\# where all voxels w/ intensity $1=$ left-right ;

intensity 2 =front-back; intensity 3 = up-down

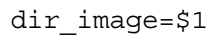

\# get distance to each principal direction echo "measuring..."

\$FSLDIR $\} /$ bin/fslmaths $\$\{$ dir_image $\}-a b s$ tmpa \# $\mathrm{x}$-dir

$\$\{$ FSLDIR $\} /$ bin/fslmaths tmpa -sub xim-sqr tmpx $\$\{$ FSLDIR $\} / \mathrm{bin} / \mathrm{fslmaths}$ tmpx -Tmean -mul 3 tmpx $\$\{$ FSLDIR $\} /$ bin/fslmaths tmpx -sqrt tmpx mv tmpx.nii.gz closex.nii.gz

$\# \mathrm{y}$-dir

$\$\{$ FSLDIR $\} /$ bin/fslmaths tmpa -sub yim -sqr tmpy $\$\{$ FSLDIR $\} /$ bin/fislmaths tmpy - Tmean - mul 3 tmpy \$FSLDIR $\} /$ bin/fslmaths tmpy -sqrt tmpy mv tmpy.nii.gz closey.nii.gz

\# z-dir

\$FSLDIR $\} /$ bin/fslmaths tmpa -sub zim-sqr tmpz $\$\{$ FSLDIR $\} /$ bin/fslmaths tmpz -Tmean - mul 3 tmpz \$FSLDIR $\} /$ bin/fslmaths tmpz -sqrt tmpz mv tmpz.nii.gz closez.nii.gz

\# combine and get index of closest direction echo "making..." $\$\{$ FSLDIR $\} /$ bin/fslmerge - $t$ xyz closex closey closez $\$\{$ FSLDIR $\} /$ bin/fslmaths xyz -mul -1 -Tmaxn -add \$mask_image $\mathrm{xyz}$

rm-rf closex.nii.gz closey.nii.gz closez.nii.gz tmpa. nii.gz arg.nii.gz argo.nii.gz xim.nii.gz yim.nii.gz zim.nii.gz echo "done!" 


\section{University Library}

\section{- M I N E R VA}

\section{A gateway to Melbourne's research publications}

Minerva Access is the Institutional Repository of The University of Melbourne

\section{Author/s:}

Alexander, B;Yang, JY-M;Yao, SHW;Wu, MH;Chen, J;Kelly, CE;Ball, G;Matthews, LG;Seal, ML;Anderson, PJ;Doyle, LW;Cheong, JLY;Spittle, AJ;Thompson, DK

Title:

White matter extension of the Melbourne Children's Regional Infant Brain atlas: M-CRIB-WM

Date:

2020-02-21

\section{Citation:}

Alexander, B., Yang, J. Y. -M., Yao, S. H. W., Wu, M. H., Chen, J., Kelly, C. E., Ball, G., Matthews, L. G., Seal, M. L., Anderson, P. J., Doyle, L. W., Cheong, J. L. Y., Spittle, A. J. \& Thompson, D. K. (2020). White matter extension of the Melbourne Children's Regional Infant Brain atlas: M-CRIB-WM. HUMAN BRAIN MAPPING, 41 (9), pp.2317-2333. https:// doi.org/10.1002/hbm.24948.

Persistent Link:

http://hdl.handle.net/11343/244178

\section{License:}

CC BY 